\title{
De la main à la production industrielle en série
}

L'articulation entre art et technique dans l'art contemporain envisagée à partir du concept de trace

\section{Cécile Dazord}

\section{(2) OpenEdition \\ Journals}

Édition électronique

URL : https://journals.openedition.org/tc/7574

DOI : $10.4000 /$ tc. 7574

ISBN : 0248-6016

ISSN : 1952-420X

Éditeur

Éditions de l'EHESS

\section{Édition imprimée}

Date de publication : 24 décembre 2015

Pagination : 124

ISBN : 978-2-7132-2505-5

ISSN : 0248-6016

Référence électronique

Cécile Dazord, «De la main à la production industrielle en série », Techniques \& Culture [En ligne], 64

2015, mis en ligne le 24 mars 2016, consulté le 29 septembre 2022. URL : http://

journals.openedition.org/tc/7574; DOI : https://doi.org/10.4000/tc.7574

Ce document a été généré automatiquement le 29 septembre 2022.

Tous droits réservés 


\section{De la main à la production industrielle en série}

L'articulation entre art et technique dans l'art contemporain envisagée à partir du concept de trace

\section{Cécile Dazord}

\section{NOTE DE L'ÉDITEUR}

Nous vous invitons à consulter la synthèse de cet article (édition papier) en téléchargeant le document placé en annexe.

\section{NOTE DE L'AUTEUR}

Ce texte a été produit dans le cadre d'un séminaire dirigé par Xavier Guchet à l'UFR de philosophie de Paris-1 sur la traçabilité. 


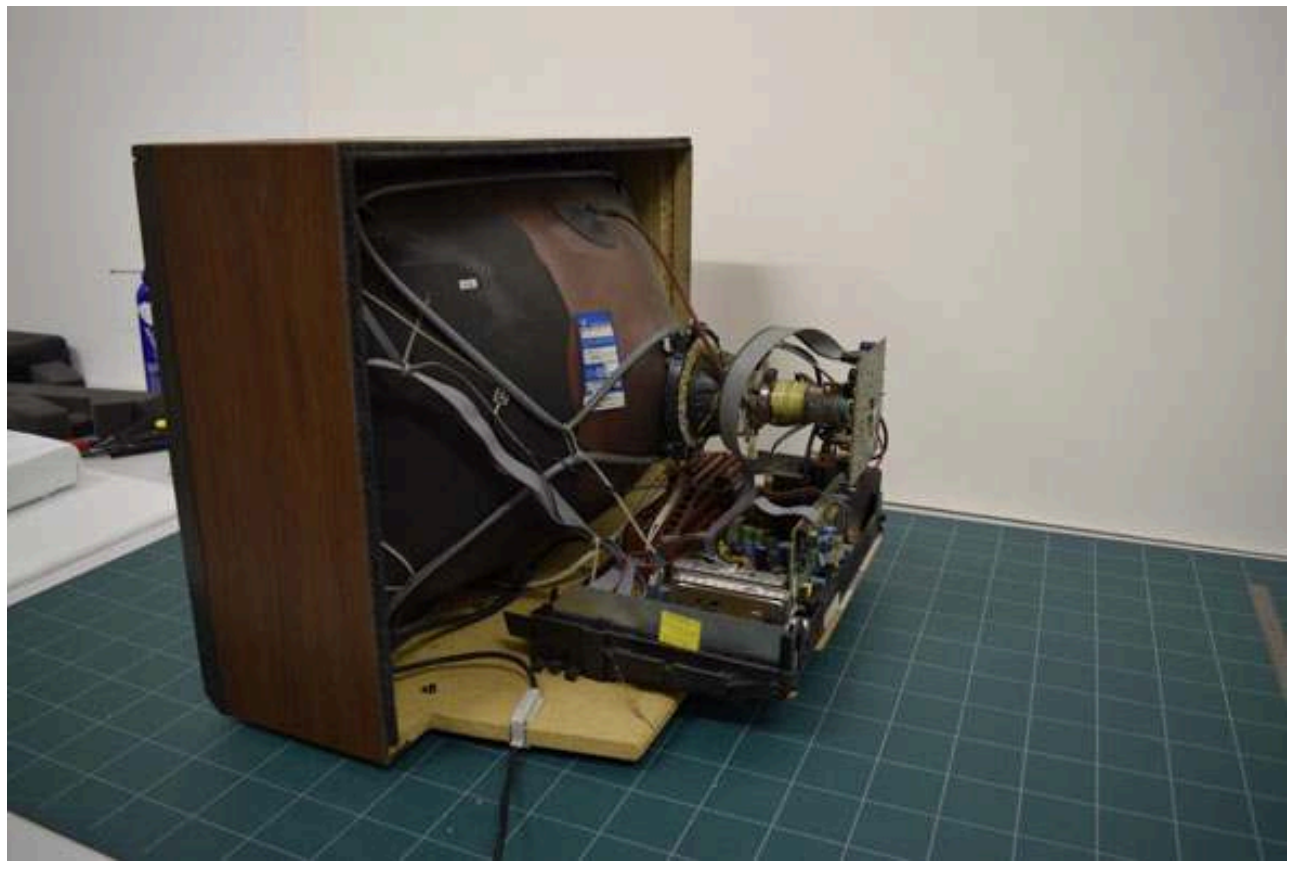

Moniteur à tube cathodique démonté, vue arrière

(C) Cécile Dazord, C2rmf

Une caractéristique de l'œuvre d'art est qu'elle procède de deux ordres, l'intelligible et le sensible, dont l'articulation conditionne les modalités de cette dernière comme vecteur de connaissance. Comment les beaux-arts traditionnels (peinture, sculpture, dessin essentiellement), fondés sur l'expression ou la traduction d'une intuition ou d'un concept dans un matériau ou une forme, puis, à partir du début $d u x^{e}$ siècle, sur des pratiques artistiques marquées par l'irruption et l'intégration d'objets produits industriellement en série, s'accommodent-ils de cette hétérogénéité sans se dissoudre dans l'extériorité du matériau ou de l'objet sériel ? Envisagé comme indice actuel et circonstancié d'un donné originaire chez Ginzburg (2010 [1986]) ou dépourvu, au contraire, de toute fonction indiciaire chez Derrida (2009 [1967], 1967), le concept de trace constitue un point de départ à partir duquel repenser le rapport entre l'intelligible et le sensible dans l'œuvre d'art et notamment la place et le statut des objets produits industriellement en série. Il s'agit, en d'autres termes, à partir du concept de trace - et de ce qui permet de le confondre avec l'empreinte, le signe, le symbole ou, au contraire, de l'en distinguer - de reconsidérer l'articulation entre l'art et la technique.

\section{L'intention artistique et le paradigme indiciaire dans les beaux-arts}

Une difficulté pour approcher et cerner les œuvres d'art (produire un discours historique, critique ou esthétique) réside dans le dualisme ou la polarité (fruit d'une longue tradition idéaliste marquée notamment par Platon, Kant, Hegel et Croce) entre l'intention ou l'intuition de l'œuvre et sa réalisation concrète. La formulation des termes de cette polarité varie selon les époques: idée, disegno, kunstwollen, intention, intuition, concept... versus matière, matériau, forme, mise en forme, manifestation 
objective, extériorisation... Un trait dominant et caractéristique de cette représentation duelle est le postulat d'une antériorité et d'une priorité du concept/idée sur la forme/ matière ; d'où résulte celui d'une indépendance du concept ou de l'idée par rapport à la forme ou à la matière. Ce schéma, fondateur des beaux-arts et de leur autonomie, est particulièrement prégnant.

Un effort pour surmonter cette dichotomie traverse l'histoire des beaux-arts: il consiste à identifier ou instaurer une continuité entre la conception d'une œuvre et sa manifestation afin de garantir une cohésion entre la singularité d'une intention (immatérielle, ineffable) et son expression ou sa traduction dans un matériau (physique, concret). L'enjeu, plus précisément, au cours de ce processus d'extériorisation, est de sauver le caractère exceptionnel de la création artistique, de distinguer le geste artistique de la fabrication artisanale. Par contamination, le geste créateur et l'objet artistique qui en résultent se trouvent affectés par la singularité de l'intention artistique; le geste artistique et l'œuvre d'art créée sont ainsi également placés sous le sceau d'une unicité irréductible. La main ou le geste artistique (dont le singulier fait écho à la singularité de l'intention) constituent ainsi un prolongement de l'intuition de l'artiste et confèrent à l'œuvre son statut d'unicum, qui la distingue radicalement de l'objet artisanal.

« Il est des objets si complexes par leurs matériaux et leurs formes qu'aucune tentative pour les reproduire ne peut dupliquer ces caractéristiques tenues pour essentielles : c'est le cas d'un tableau à l'huile exécuté avec des couleurs particulières sur une toile particulière, de manière que les ombres, la structure de la toile et les coups de pinceau, tous éléments essentiels à la jouissance du tableau comme œuvre d'art, ne peuvent jamais être totalement reproduits. [...]. La notion moderne d'œuvre d'art comme irreproductible et unique assigne un statut spécial tant à l'origine de l'œuvre qu'à sa complexité formelle et matérielle, qui constituent ensemble le concept d'authenticité autorielle. » (Eco 2010 [1990] : 182)

2 L'instauration d'une continuité entre une intention et son extériorisation par le truchement de la main ou du geste scelle un pacte: dans la matière ou la facture de l'œuvre se trouve consignée la marque (unique et non reproductible) de l'artiste. L'œuvre d'art, qui porte ainsi l'empreinte ou la trace de la singularité de la main ou du geste de l'artiste, peut dès lors naturellement être livrée à l'œil (tout aussi unique et singulier) du connaisseur ou de l'expert. Dans cette perspective, une observation attentive de l'œuvre permet une identification de l'artiste, autrement dit une authentification. En d'autres termes, l'histoire des beaux-arts très largement fondée, sinon sur le seul attributionnisme, du moins sur la reconnaissance et l'individualisation des styles à partir de l'examen des œuvres jusque dans leurs moindres détails, procède $\mathrm{du}$ « paradigme sémiotique ou indiciaire » mis en évidence par Carlo Ginzburg (ibid.).

3 L'empreinte procède de la main ou du geste, autrement dit du corps de l'artiste. L'instauration d'une distance croissante, tout au long $\mathrm{du} \mathrm{xx}^{\mathrm{e}}$ siècle, entre l'artiste et l'exécution de son œuvre (déléguée à un tiers ou laissée au hasard, remplacée par l'intégration pure et simple d'un objet ou par un procédé mécanique), n'a pas pour autant révoqué ce paradigme. À défaut de la main, le geste et le corps de l'artiste ont ressurgi de la peinture de Jackson Pollock qui semblait avoir réglé son compte à la facture manuelle en réduisant l'application de la peinture sur la toile à des jets et coulées pratiqués à partir des pots. L'engouement pour les photographies et les films de 
Hans Namuth le montrant au travail ont conduit à une réinterprétation de ses toiles placées sous le signe de la performance dont la peinture constitue alors une trace. Le ready-made ou le Pop art n'ont réduit ni la visibilité, ni l'ascendant d'artistes tels que Marcel Duchamp ou d'Andy Warhol, bien au contraire. Quant à l'art de la performance, qui se développe à partir de la fin des années 1960, il repose précisément sur l'implication du corps de l'artiste dans l'œuvre. Le paradigme indiciaire résiste même à l'introduction de la reproduction mécanisée. Se situant explicitement dans une perspective sémiotique, Rosalind Krauss emprunte à Charles Peirce sa définition de l'indice et énonce le caractère indiciel de la photographie - alors exclusivement analogique :
«Toute photographie est le résultat d'une empreinte physique qui a été transférée sur une surface sensible par les réflexions de la lumière. La photographie est donc le type d'icône ou de représentation visuelle qui a avec son objet une relation indicielle. » (Krauss 1993 [1977] : 69)

On peut invoquer ici la distinction instaurée par Nelson Goodman (avant d'être reprise par Gérard Genette) entre arts " autographiques » et " allographiques », les premiers se distinguant des seconds par leur dépendance à l'égard des processus de production et d'exécution. On peut voir dans cette notion de dépendance une autre formulation du paradigme indiciaire. Significativement, ce distinguo est associé chez Goodman à la possibilité ou non de la contrefaçon soit, corollairement, à la problématique de l'authenticité et de l'authentification - elle-même étroitement liée à celle de l'unicité de l'œuvre d'art :

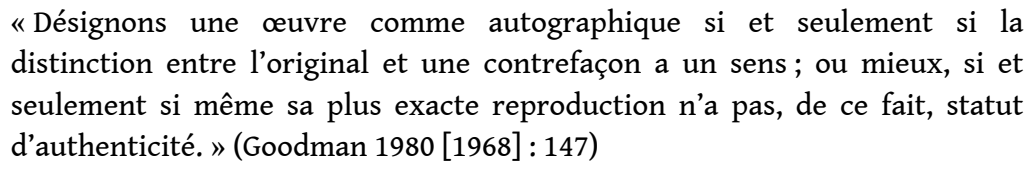

Même si ses racines sont anciennes et remontent à la chasse, Ginzburg date l'apparition du paradigme indiciaire de la fin du XIX ${ }^{e}$ siècle qui coöncide précisément avec l'âge d'or de l'attributionnisme : "vers la fin du xix ${ }^{\mathrm{e}}$ siècle - plus précisément dans la décennie 1870-1880 - commença à s'affirmer dans les sciences humaines un paradigme indiciaire fondé précisément sur la sémiotique.» (ibid: 233) Cette coïncidence temporelle avec l'essor de l'attributionnisme n'est pas anodine: le paradigme indiciaire a partie liée avec l'idée d'authenticité et, surtout, recèle les conditions de possibilité de l'authentification. Parallèlement, des faux de Vermeer par van Meegeren (1889-1947) à la retentissante " Affaire Beltracchi ${ }^{1}{ }^{»}$ (Koldehoff, Timm 2013), les exemples d'experts abusés ne manquent pas. Si le paradigme indiciaire apparaît extrêmement prégnant dans le domaine des beaux-arts, au point non seulement de gouverner les représentations, mais de guider et orienter les pratiques, il n'en demeure pas moins fragile, bien qu'il ait été considérablement renforcé, depuis le début $\mathrm{du} \mathrm{xx}^{\mathrm{e}}$ siècle, par les apports de la physique chimie et de l'imagerie scientifique à l'analyse des œuvres d'art.

« En termes de langage naturel, nous savons tous peu ou prou ce que sont un faux et une contrefaçon. On admet en général qu'il est souvent difficile de reconnaître un faux, mais on se fie à des experts, lesquels sont en mesure de reconnaître les contrefaçons, pour la bonne raison qu'ils savent comment déceler la différence entre un faux et un original. En fait, les définitions de 
termes comme faux, contrefaçons, pseudépigraphe, falsification, fac-similé, illégitime, pseudo, apocryphe et autres, sont plutôt controversées. Il est raisonnable de penser que les difficultés à définir ces termes tiennent aux difficultés à définir la notion même d'"original" ou d'"objet authentique". " (Eco : 175)

6 Ce que rend manifeste la problématique du faux c'est que la tromperie ou la duperie ne relève pas d'une propriété intrinsèque de l'objet, mais consiste dans l'énonciation d'une attribution erronée ou usurpée. Le faux rend ainsi tangible la fragilité du paradigme indiciaire dans les beaux-arts - du postulat d'un lien de causalité entre une œuvre et l'intentionnalité dont elle procède et de la possibilité, a contrario, à partir de l'œuvre et de son observation attentive et détaillée, de remonter par abduction ou inférence à son référent ou à son origine. En ce sens, l'introduction dans l'art contemporain d'objets qui ne sont plus exécutés de la main d'un artiste et ne constituent plus des réalisations uniques remet en jeu, du même coup, la question de l'authenticité et le paradigme sémiotique ou indiciaire.

\section{Intégration, dans les œuvres d'art, d'objets produits industriellement en série}

\section{Inadéquation du paradigme indiciaire}

Du ready-made au Pop art, le $\mathrm{xx}^{\mathrm{e}}$ siècle se caractérise notamment par l'intégration pure et simple d'objets dans l'art. La question qui se pose alors est: comment éviter une dissolution de l'art (original et unique) dans l'objet (anonyme et multiple) ? L'enjeu pour sauver la part artistique consiste à cerner ce qui caractérise l'art, soit à saisir ce qui le distingue de l'artisanat (savoir faire) puis de la technique à l'ère industrielle (machine). La confrontation entre l'art et la technique n'est pas inédite, loin s'en faut. Dès la Renaissance, c'est précisément en se distinguant de l'artisanat qu'émergent les beaux-arts. L'introduction massive dans l'art au cours $\mathrm{du} \mathrm{xx}^{\mathrm{e}}$ siècle d'objets produits industriellement en série ne fait que donner une acuité et une tonalité nouvelles à cette problématique. À la répétabilité de l'objet artisanal et à la sérialité de l'objet produit industriellement, s'opposent la singularité et l'unicité de l'œuvre d'art. Plus précisément, alors que l'objet (artisanal ou industriel - sur ce point, la différence importe peu) consiste en une multiplicité d'occurrences interchangeables procédant d'un même type, dans l'œuvre d'art « un objet unique devient un type » (ibid: 182). On perçoit dès lors combien l'introduction dans l'art, ainsi fondée sur la confusion ou la collusion entre l'occurrence et le type, d'objets artisanaux ou industriels rigoureusement distincts du type dont ils sont issus et caractérisés par leur répétabilité ou reproductibilité peut brouiller les pistes; combien la présence d'objets sériels semble ainsi contrevenir au paradigme indiciaire qui sous-tend les beaux-arts.

7 De toute évidence, l'introduction de l'objet sériel dans les œuvres d'art n'a pas aboli les notions - étroitement liées - d'unicité et d'authenticité. Les pratiques liées à la circulation des œuvres (musée, collection, marché) suffisent à en témoigner. En dernier recours et de manière profondément tautologique, l'unicité et l'authenticité d'une œuvre se résument à sa limitation à une seule occurrence dont le numéro d'inventaire constitue la traduction (même si l'objet qui la constitue est reproductible ou interchangeable), - voire à plusieurs occurrences strictement incluses dans une série 
limitée et numérotée. Initialement, la pratique de la série limitée et numérotée peut être comprise dans une perspective sémiotique ou indiciaire : une série procède d'une matrice à partir de laquelle plusieurs occurrences sont engendrées; la numérotation renvoie au degré de proximité ou d'éloignement de l'occurrence par rapport à la matrice. Courante pour identifier la fonte d'un bronze par rapport à un moule ou le tirage d'une photographie argentique par rapport à un négatif, l'accroissement du numéro dans la série désigne alors une perte de génération, une qualité moindre par rapport à la matrice. En réalité, selon cette acception stricte, la pratique de la série limitée et numérotée ne rompt pas avec le principe de l'unicité de l'œuvre d'art fondée sur une corrélation stricte entre l'unicité de l'intention et celle de sa manifestation physique ; elle transpose simplement l'unicité de la matérialité de l'œuvre à celle de la matrice dont peuvent procéder plusieurs sous-occurrences, limitées en nombre et distinguées selon leur degré de proximité avec cette dernière. Le principe d'un référent d'origine, produit directement par l'artiste et dont l'œuvre découle est maintenu.

La pratique de la série limitée et numérotée s'est toutefois généralisée en art contemporain et étendue à des techniques reposant sur la reproductibilité mais dépourvues du phénomène de perte de génération comme la vidéo numérique pour ne citer que cet exemple. Par extension et contamination, elle est même appliquée à des procédés ne reposant pas sur la reproductibilité - plusieurs versions (rigoureusement identiques ou seulement semblables, selon les cas) d'une installation réalisée par un artiste et numérotées (trois occurrences généralement) ${ }^{2}$. La limitation et la numérotation répondent alors explicitement à une logique extrinsèque à l'œuvre et liée à celle de la collection et du marché. Héritée du paradigme indiciaire ou sémiotique, la série limitée et numérotée traduisait initialement un degré de proximité et d'authenticité par rapport à un référent. En art contemporain, elle apparaît parfois purgée de toute référence à une entité matricielle ou originelle dont elle procéderait, rompant ainsi avec le principe du paradigme indiciaire ou sémiotique. Ce que traduit, en définitive, cette pratique de la série numérotée et limitée sans référent originel ou matriciel, c'est l'absence de continuité - physique - entre l'œuvre et l'intention dont elle procède. L'objet produit en série et présent dans une œuvre d'art contemporain ne recèle pas nécessairement en lui la marque ou l'empreinte d'un geste créateur.

Toute la question consiste désormais à articuler l'unicité de l'œuvre d'art à la sérialité de l'objet rigoureusement interchangeable, indéfiniment reproductible. Si l'horizon temporel d'une œuvre d'art dans un cadre patrimonial est a priori illimité, il n'en va pas de même de la durée de vie des objets. À ce point, interviennent alors les notions corollaires - cruciales et déterminantes - de consommable et de fonctionnement. Parmi les objets produits en série et intégrés dans les œuvres contemporaines certains constituent des consommables, autrement dit recèlent des ressources énergétiques qui produisent un fonctionnement mais s'épuisent dans le temps. C'est le cas d'une ampoule électrique dans une œuvre de Christian Boltanski ou d'un moteur dans une installation de Jean Tinguely (1925-1991). Or la lumière ou le mouvement ne sont pas, dans les deux cas mentionnés, strictement fonctionnels: ils ne constituent pas des accessoires permettant de voir et percevoir les œuvres, mais sont destinés à être vus et perçus comme tels. Sachant que la durée de vie des ampoules et des moteurs est nécessairement inférieure à celle de la durée de conservation de ces œuvres dans une institution patrimoniale, la question de la maintenance du fonctionnement et de ses modalités se pose tout naturellement. 
10 En pratique, lorsque la substitution à l'identique d'un consommable est possible, elle est effectuée. Ce type d'opération est mené malgré l'absence de socle théorique et de codification des pratiques de restauration pour les œuvres contemporaines - la publication de la Teoria del restauro de Cesare Brandi remonte à 1963; la prise de conscience dans le cadre de réseaux internationaux para-institutionnels d'une spécificité des problématiques conservatoires dans le champ de l'art contemporain remonte seulement aux années 2000 et n'a pas à ce jour donné lieu à un corpus de référence. Dans une œuvre de Christian Boltanski, les ampoules sont changées sans que l'authenticité de l'œuvre soit perçue comme affectée par cette opération. Plusieurs principes implicites sont sous-tendus par cette substitution à l'identique: le fonctionnement est considéré comme déterminant; l'absence de continuité physique entre l'objet en série et l'intentionnalité artistique est reconnue et autorise, précisément, la substitution. Dans la pratique, toujours, (puisque la conservationrestauration des œuvres contemporaines est essentiellement régie par la pratique), les responsables de collection tendent à se couvrir et à légitimer les substitutions - pour le moins hétérodoxes au regard de la théorie de la restauration et des pratiques en vigueur, solidement arrimées à un principe d'équivalence strict entre l'unicité de l'œuvre d'art et l'unité de sa matérialité, proscrivant toute forme de substitution - en faisant appel à l'artiste et à son intention, sous la forme généralement d'une parole recueillie et consignée comme une caution, visant à garantir en quelque sorte l'opération ${ }^{3}$. Ce recours à la formulation verbale d'une intentionnalité artistique est paradoxal: il traduit, d'un côté, la rupture de continuité entre l'intentionnalité artistique et la matérialisation de l'œuvre ; de l'autre la résistance du credo selon lequel la matérialisation de l'œuvre procède d'une intentionnalité qui existe indépendamment et la précède, qu'il suffit d'extraire et qui constitue comme un code source (unique et invariant) de l'œuvre. À la main ou au geste de l'artiste s'est substituée la parole - dans lequel le paradigme indiciaire peut encore se loger. Face à la sérialité et à l'interchangeabilité de l'objet qui déstabilise l'unicité de l'œuvre, l'invocation - via la parole recueillie - de l'intentionnalité artistique singulière et immuable constitue un moyen de re-fonder ou re-légitimer l'unicité de l'œuvre d'art.

11 L'art contemporain, dans la pratique, s'est donc accommodé de la présence d'objets fabriqués industriellement dans les œuvres, de la reproductibilité et de l'interchangeabilité des objets d'une part et de l'unicité irréductible des œuvres d'autre part. Aujourd'hui pourtant, l'objet sériel substituable à l'identique semble appartenir à une époque révolue. À rebours de l'horizon infini qu'évoquait la reproductibilité mécanisée et qui sous-tend l'«Antiquiertheit des Menschens» - "l'obsolescence de l'homme » - formulée par Günther Anders (2002 [1956]), l'augmentation effrénée des phénomènes d'obsolescence dans tous les secteurs a drastiquement réduit, non seulement la durée de fonctionnement des objets au sein d'une série, mais également et surtout la durée de vie des séries, types ou générations d'objets eux-mêmes et, ce faisant, profondément modifié leur place et leur inscription dans la société. Contrairement à une obsolescence dite "programmée » qui désigne un raccourcissement du temps de fonctionnement des consommables et qu'il serait plus juste, en définitive, de nommer usure programmée (Slade 2006), l'« obsolescence technique " ou "obsolescence par incompatibilité " qui affecte véritablement la conservation et la restauration des œuvres contemporaines, se joue non pas au niveau de l'occurrence mais au niveau du type : une classe entière d'objets est révoquée des circuits de distribution et de production et remplacée par une autre présentée comme 
plus performante. Le bannissement des ampoules à incandescence - présentées comme trop énergivores - dans divers pays du globe depuis le début des années 2000 (les calendriers et réglementations varient selon les pays) en constitue un parfait exemple.

\section{L'obsolescence et la part technique des œuvres}

\section{Le concept de trace pour envisager l'articulation entre art et technique}

L'obsolescence, en introduisant un coup d'arrêt dans une série illimitée, a pour double conséquence d'inscrire l'objet dans une temporalité circonscrite et de le raréfier. Au régime temporel complexe de l'œuvre (constitué du moment de sa création et des divers moments de sa réception au cours du temps et volontiers conçu comme écart par rapport à son temps) se superpose celui de l'objet, produit circonstancié de son temps, dont il constitue un marqueur. À la singularité et à l'originalité de l'œuvre, s'ajoute la rareté de l'objet qui concentre sur lui une attention qu'il n'attirait pas lorsque la série avait cours. Cette introduction, dans l'œuvre, par le biais de l'objet obsolète, d'informations et de connotations éventuellement étrangères à l'intentionnalité de l'artiste fait de l'objet sériel présent dans l'œuvre d'art et, par extension, de l'œuvre elle-même, un indice ou une trace au sens où l'envisage Derrida (ibid), déconnecté de toute intention de signification préalable («Bedeutung»). S'employant à renverser le paradigme qui consiste à concevoir l'écriture comme extériorisation du logos autrement dit de la pensée et de l'oralité intimement liées l'une à l'autre -, comme seconde par rapport à un logos, préexistant, Derrida reconsidère toute la théorie du signe (ses racines "métaphysico-théologiques", la dichotomie entre l'âme et le corps) pour aboutir à la formulation d'un concept de "trace " ne procédant pas d'un " vouloir-dire » préconstitué et se construisant dans la différence. Le rapport analysé et déconstruit entre le logos et l'écriture présente de nombreuses similitudes avec le lien entre l'intuition et l'œuvre réalisée. Comme l'écriture, l'œuvre d'art mérite donc d'être soustraite au paradigme sémiotique et d'être pensée comme trace - distincte du signe ou de l'indice.

En différant l'objet, qui procède désormais d'une temporalité révolue, l'obsolescence fonctionne comme un révélateur. Ce que l'impossibilité de substitution à l'identique rend manifestes ce sont moins des propriétés intrinsèques que les différentes modalités selon lesquelles un objet (ou une technique) est intégré dans une œuvre d'art et l'affecte ou la contraint - en d'autres termes, son statut ou son rôle. Dans une performance filmée, on peut considérer que la vidéo est intégrée de manière strictement fonctionnelle, autrement dit qu'elle est insignifiante, indifférente, sans incidence. Pourtant, la vidéoprojection qui apparaît dans les années 1980 permet de donner à voir des images sans passer par le truchement du moniteur - qui connote immanquablement la télévision - ce qui jusqu'alors était rigoureusement impossible. La technique qui pouvait sembler accessoire, apparaît manifestement - dès lors qu'elle devient obsolète - porteuse de significations distinctes des techniques proposées en remplacement. Au contraire, certaines œuvres apparaissent totalement dépendantes d'une technique au point qu'elles ne semblent pas pouvoir être activées si cette technique n'est plus disponible : c'est le cas notamment de Magnet TV de Nam June Paik, 
présentée à Wüppetal en 1962, qui consiste en un aimant disposé sur un moniteur à tubes cathodiques (CRT de l'anglais : cathode ray tube).

Tube cathodique, vue arrière, détail (canon à électrons)

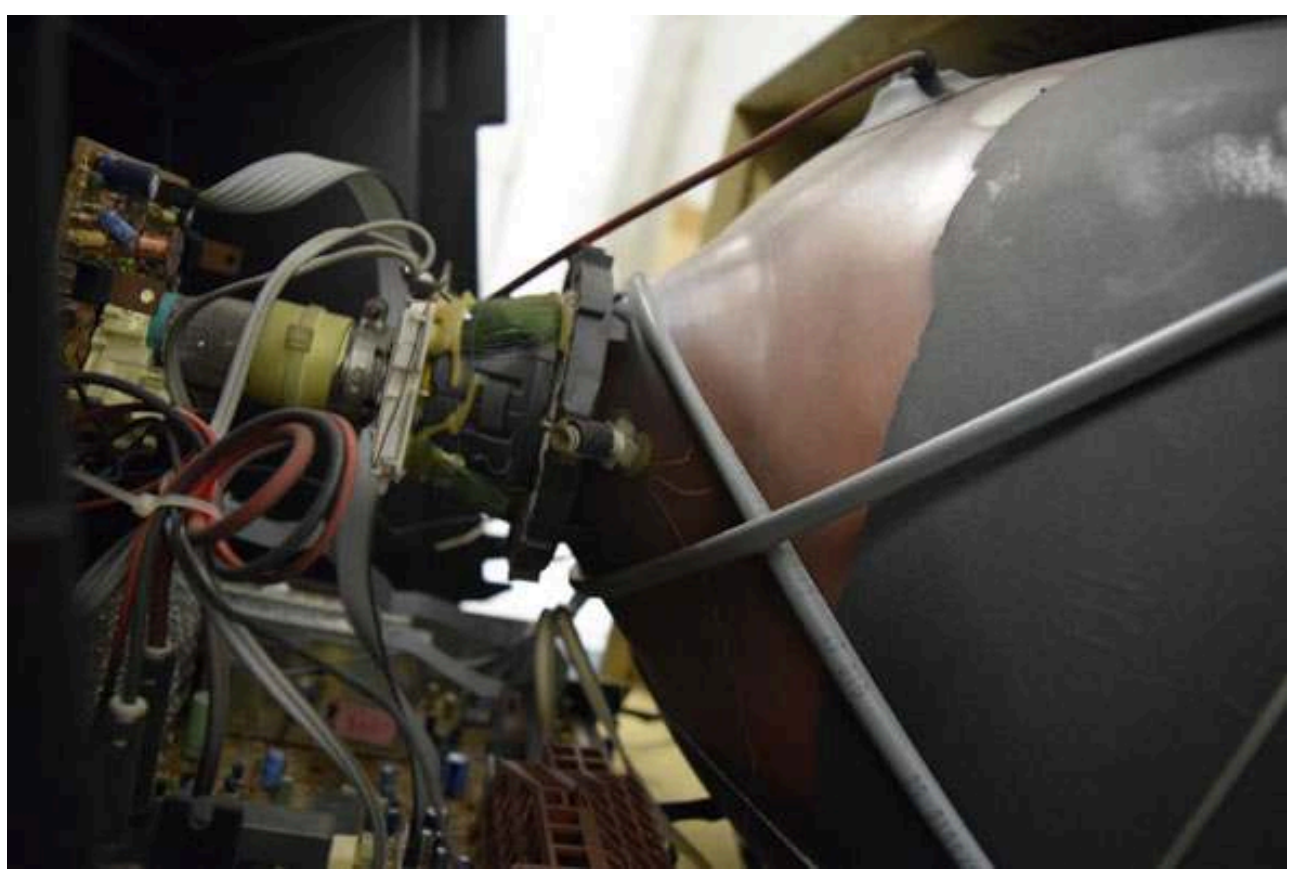

(C) Cécile Dazord, C2rmf

Vue rapprochée de l'écran sous tension (luminophores rouges, verts bleus)

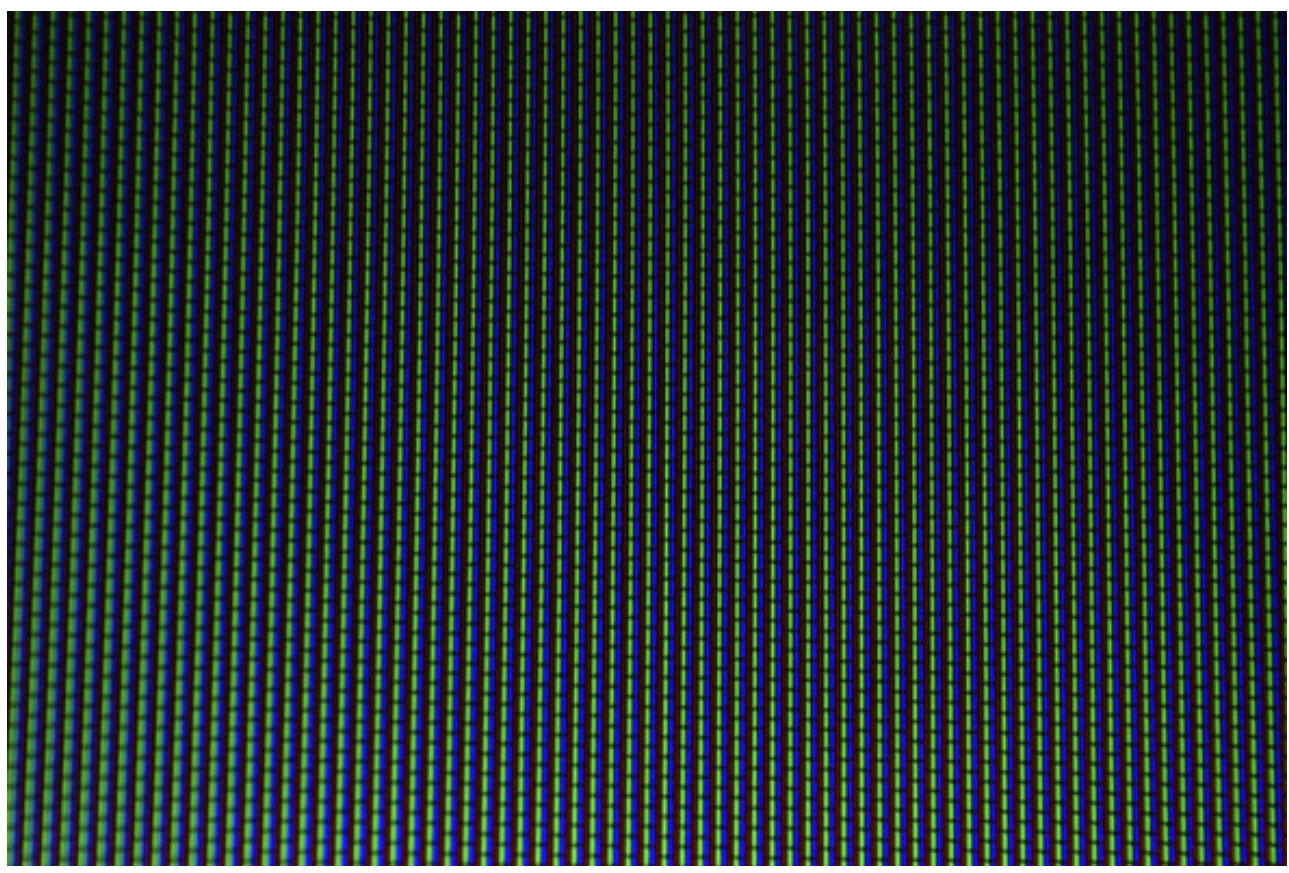

(c) Cécile Dazord, C2rmf

13 La perturbation du champ magnétique et du balayage des électrons produite par l'aimant brouille l'image diffusée en temps réel. Aux tubes cathodiques ont succédé 
d'autres technologies dont les champs magnétiques sont plus stables et qui ne permettent pas de reproduire cet effet. Dans ce cas, l'obsolescence fait apparaitre le caractère irréductible de la technique. En l'absence de CRT (ils ne sont plus disponibles dans le commerce à ce jour), l'œuvre peut être au mieux évoquée (sous forme d'une séquence enregistrée figée et passée en boucle par exemple) mais non recréée en temps réel. D'autres œuvres, enfin, n'empruntent pas un objet ou une technique (qui se trouvent ainsi décontextualisés), mais naissent avec une technique, voire en découlent ou en résultent. C'est le cas de la photographie ou du film qui, nés avec l'industrie, à la différence des beaux-arts, tolèrent mieux les évolutions techniques et se perçoivent comme "média in transition" selon la formule de William Uricchio (2004), même si chaque innovation suscite de vives résistances - il suffit de songer à l'avènement du cinéma parlant. Si la technique peut être présente dans les œuvres à divers titres, les critères qui permettent d'en décider semblent fragiles (variables avec le temps); plus ou moins intrinsèquement liée à l'œuvre, la technique ne semble cependant jamais anodine. À l'instar du concept de trace défini par Derrida, elle n'apparait pas d'emblée donnée mais au contraire variable selon les contextes.

Que l'avènement de la photographie et du film ait alimenté la réflexion sur les rapports entre l'art et la technique, cela apparaît nettement dans les écrits de Benjamin (luimême lecteur de l'ouvrage publié en 1925 par Lazlo Moholy-Nagy, Peinture film, photographie) (Passuth 1980). Outre une connaissance fine des procédés, Benjamin a une conscience extrêmement aiguë du rôle contraignant ou signifiant de la technique sur les œuvres produites. Loin d'envisager la photographie comme un domaine homogène ou indistinct, il analyse scrupuleusement les différentes techniques photographiques, opposant ainsi radicalement la photographie des premiers temps, dont les longs temps de pose conduisaient à la production d'images composées à la manière des peintures, à la photographie instantanée qui permet la saisie sur le vif.

«Les accessoires de ce genre de portraits, piédestal, balustrades et autres petites tables ovales, rappellent l'époque où la longue durée de l'exposition obligeait à fournir un point d'appui aux modèles afin qu'ils restassent immobiles. Si au début on s'était contenté d' "appuis-tête" ou de "repose genoux", suivirent bientôt d'autres accessoires semblables à ceux qui figuraient dans les peintures célèbres et qui, pour cette raison, devaient apparaître "artistiques". Ce furent d'abord les colonnes et les rideaux. Des voix avisées s'élevèrent contre ces excès dès les années 1860 . Ainsi pouvaiton lire dans une revue anglaise spécialisée: "Dans les œuvres peintes, la colonne apparaît vraisemblable, mais la façon dont on l'utilise en photographie est absurde, car elle est d'ordinaire posée sur un tapis. Or chacun sera convaincu qu'une colonne de marbre ou de pierre ne peut avoir un tapis pour fondation".» (Benjamin 2012 [1938])

Peinture, photographie et film de Laszlo Moholy-Nagy fait de la photographie et du film le creuset dans lequel s'éveillent au $x^{e}$ siècle le goût pour les jeux sur la lumière et le mouvement. À vrai dire, cela semble une évidence incontestable, proche du truisme. Pourtant une hiérarchisation encore fortement prégnante dans le domaine des beauxarts, malgré les affirmations réitérées par chaque avant garde de synthèse et de fusion des arts ou de trans- ou pluridisciplinarité, continue d'envisager la photographie ou le film en marge ou en parallèle des beaux-arts. À quelques rares exceptions près ${ }^{4}$, les grandes expositions sur le mouvement et la lumière dans l'art $\mathrm{du} \mathrm{xx}^{\mathrm{e}}$ siècle sont des expositions de peintures et de sculptures - en mouvement, lumineuses - 
éventuellement ponctuées de photographies et de films (l'exposition Dynamo récemment organisée au Grand Palais est parfaitement emblématique de cette approche générique et stylistique du mouvement et de la lumière - à partir des formes de la peinture et de la sculpture et de la problématique de l'abstraction). Rarement la photographie et le film sont présents au même titre que la peinture et la sculpture; plus rarement encore la photographie ou le film sont présentés comme les déclencheurs de ce goût et de cet engouement pour la lumière et le mouvement (qu'ils viennent au contraire ponctuer ou illustrer en marge). Que le film ou la photographie continuent d'occuper une place annexe dans l'histoire de l'introduction de la lumière ou du mouvement dans l'art en dit long sur la difficulté à appréhender précisément les objets et les techniques et leur action signifiante ou contraignante. La puissance du paradigme sémiotique ou indiciaire rend difficile une saisie de l'œuvre d'art hors de ce schéma. L'introduction d'objets sériels dans les œuvres, loin de mettre à distance la figure de l'artiste et de conduire à un examen attentif et détaillé des objets et des techniques comme traces porteuses de significations, a fait resurgir l'artiste sous la forme de l'intentionnalité (volontiers explicitement consignée). Pour citer à nouveau Benjamin :

\footnotetext{
" Ici entre en scène avec toute sa lourdeur le concept d' "art", auquel toute considération technique reste étrangère et qui sent venir sa fin avec l'apparition provocante de la nouvelle technique. C'est malgré tout avec ce concept fétichiste, fondamentalement anti-technique de l'art, que les théoriciens de la photographie, pendant presque cent ans, ont voulu polémiquer sans naturellement aboutir au moindre résultat. Car ils n'ont rien fait d'autre que justifier le photographe face à la juridiction même que celui-ci reversait. Un tout autre souffle se dégage de l'exposé avec lequel le physicien Arago défendit l'invention de Daguerre, le 3 juillet 1839, devant la chambre des députés. La beauté de ce discours tient à ce qu'il y découvre le lien entre tous les aspects de l'activité humaine. » (ibid: 10)
}

Du reste, s'agissant des œuvres cinétiques, la distinction est rarement faite par les historiens et critiques entre les différents modes d'intégration du mouvement et de la lumière dans les œuvres. Les nuances sont pourtant importantes et, là encore, significatives. Schématiquement, concernant les avant-gardes du début du siècle, puis l'art cinétique des années 1950-1960, les artistes s'approprient (détournent) la technique (les moteurs, les ampoules et les circuits) à leur échelle, celle de la fabrication humaine, du bricolage. Le mouvement est celui de la mainmise par l'art sur des objets et des outils techniques, la revendication d'un amateurisme ou d'un dilettantisme dans la mise en œuvre, une critique sous-jacente du productivisme. Les œuvres aux antipodes de l'ingénierie et au fonctionnement souvent erratique de Jean Tinguely sont emblématiques de cette tendance. Réalisée en 1984 et entrée dans les collections du centre Pompidou en 1990, L'Enfer est entièrement constituée de matériaux de récupération. Il en va tout autrement de la résurgence de l'art cinétique à partir des années 1990 dans des œuvres qui lui font explicitement référence et prennent éventuellement la forme de l'hommage. Le plus souvent, la production est entièrement industrielle, nullement assumée par l'artiste. Ce mode de réalisation, à l'échelle de la machine et non de la fabrication humaine, produit un effet diamétralement opposé, souvent spectaculaire. En 2003, l'installation lumineuse monumentale Weather project de l'artiste danois Olafur Eliasson dans la gigantesque 
halle des turbines de la Tate Modern de Londres, simulant un lever de soleil, a constitué un succès à la fois critique et public sans précédent pour l'institution.

\section{Néon en cours d'étude}

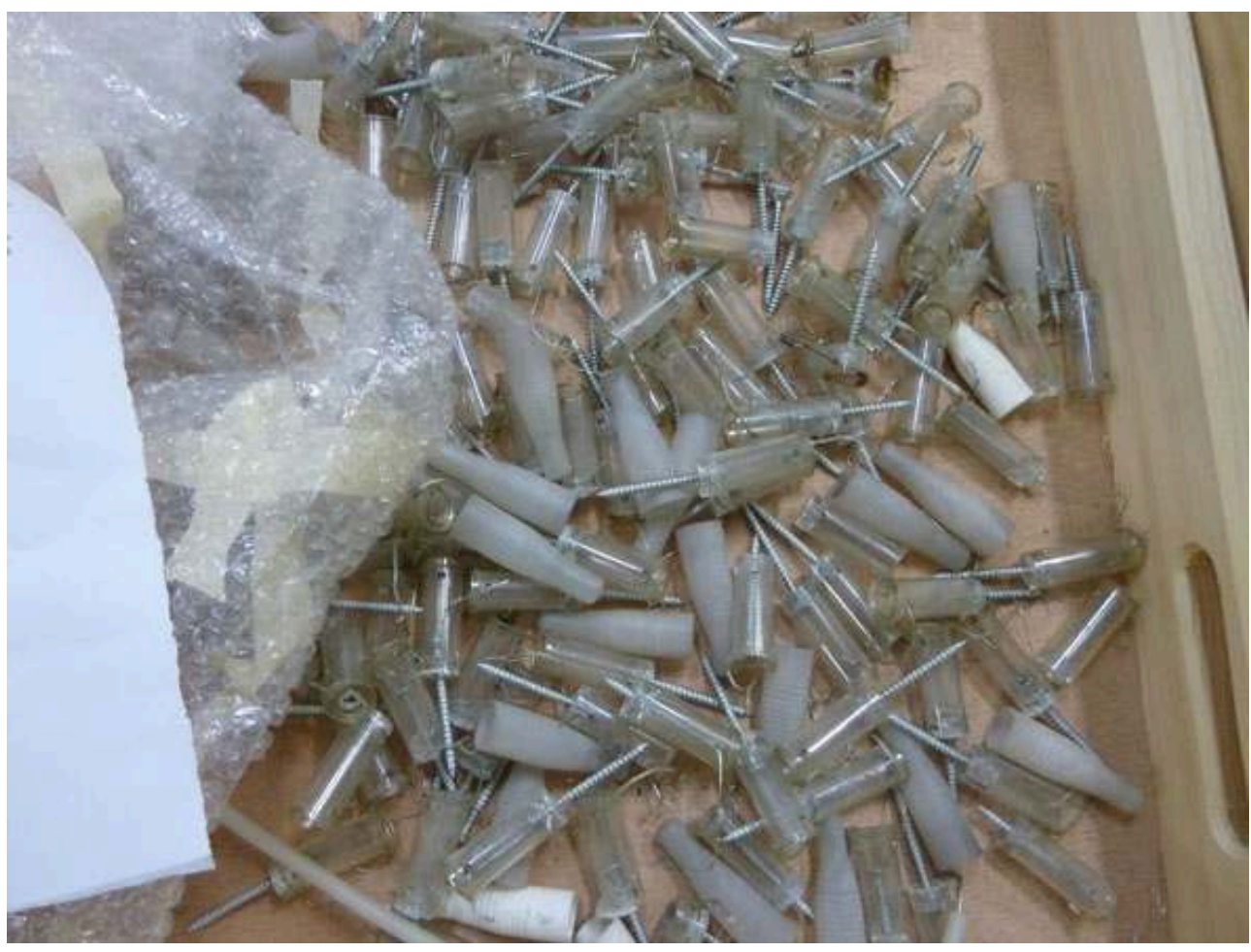

Taquets et vis utilisés pour l'accrochage. Capuchons de silicone protégeant les électrodes.

(c) Cécile Dazord, C2rmf 
Alimentation électrique : transformateurs

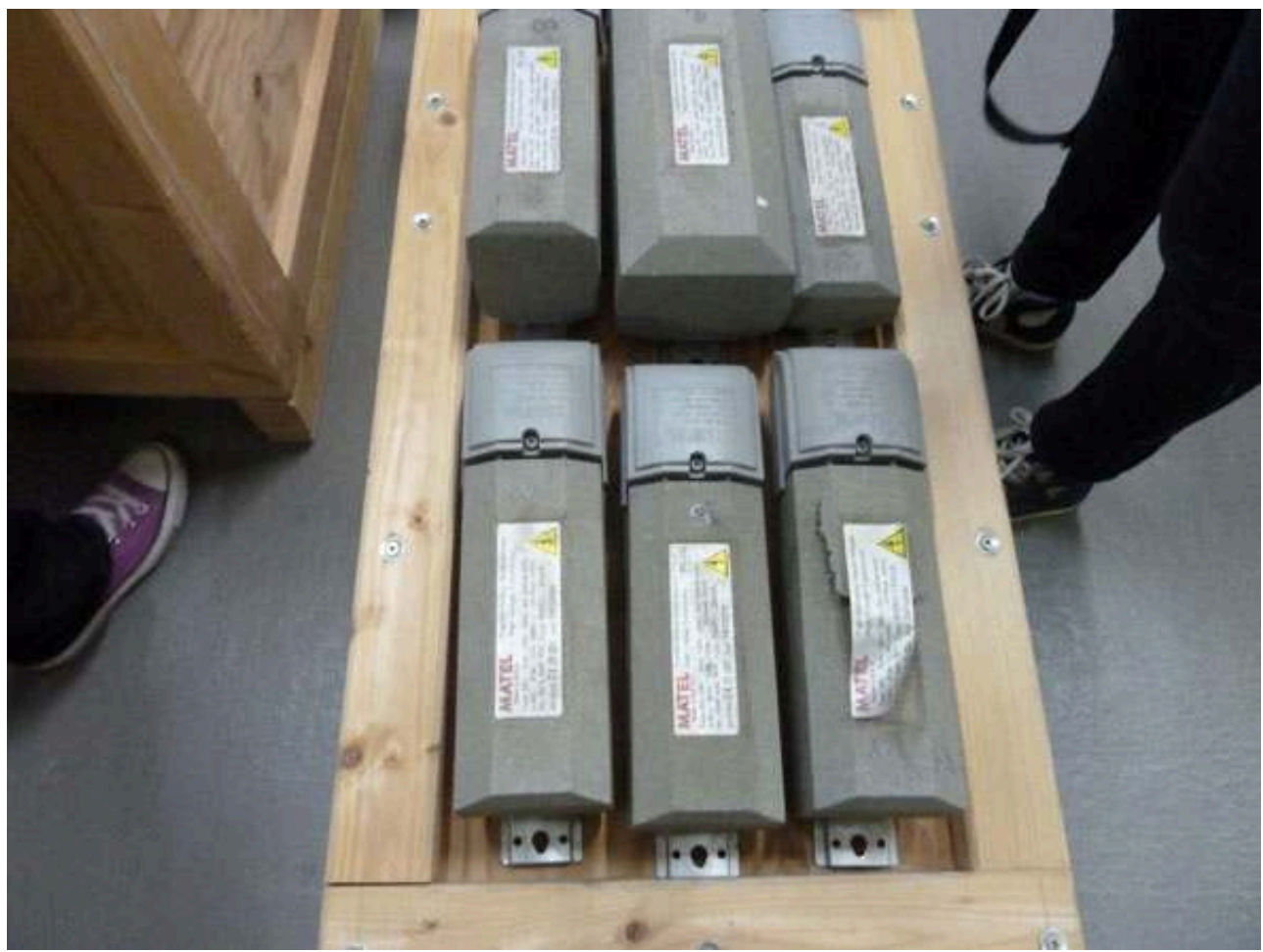

(C) Cécile Dazord, C2rmf

Test de luminosité et température de couleur

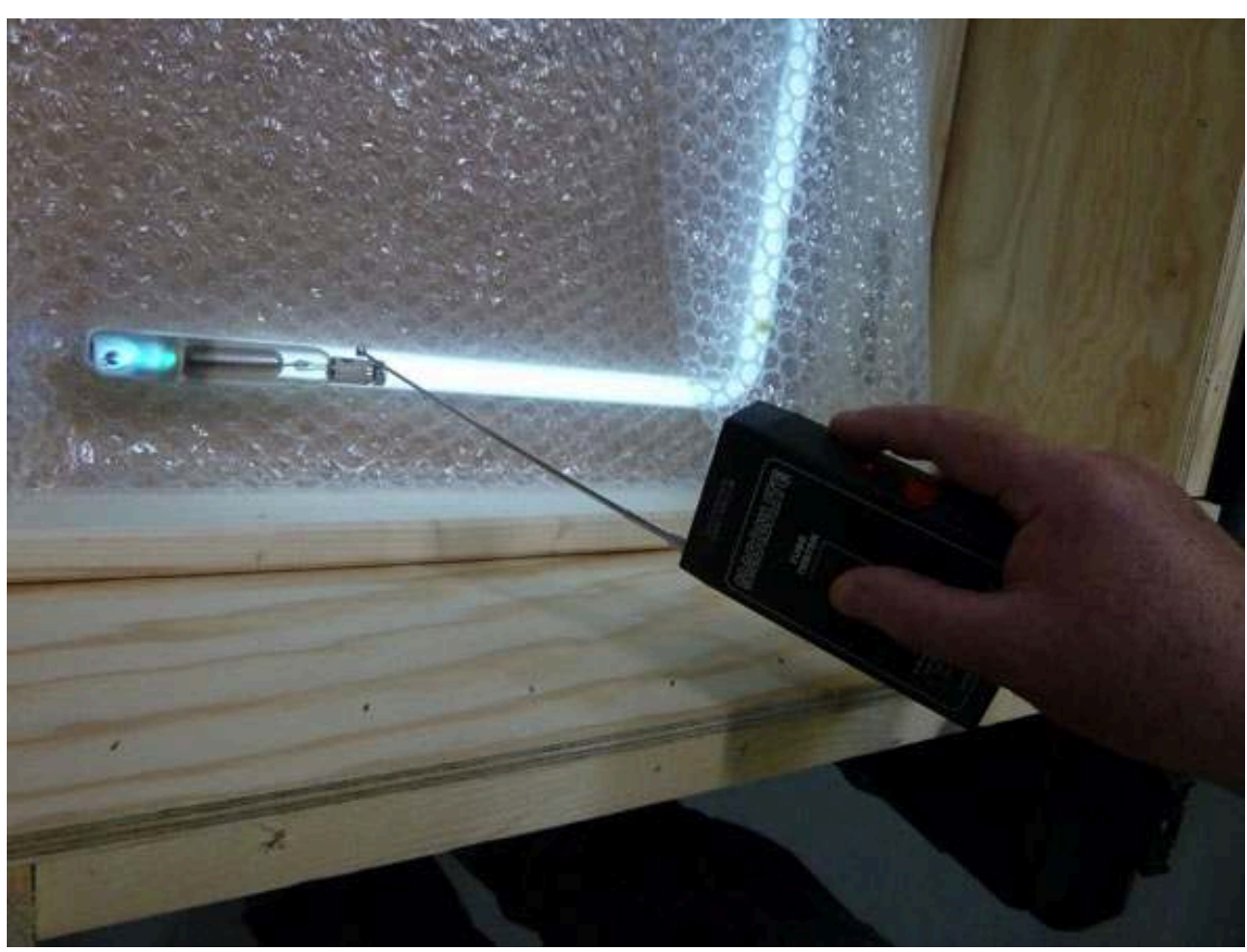

(c) Cécile Dazord, C2rmf 
Test de fonctionnement : branchement.

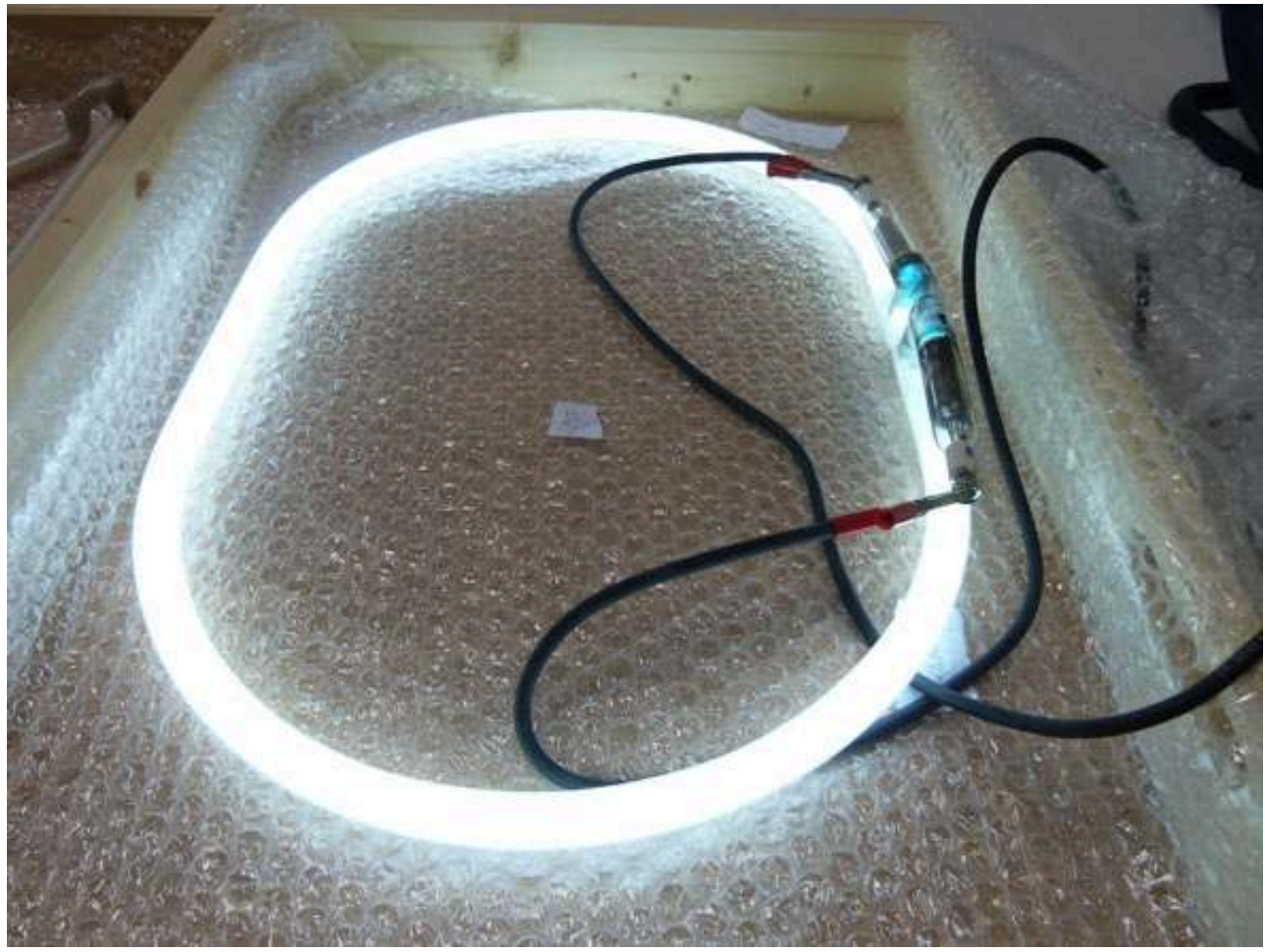

(c) Cécile Dazord, C2rmf

Test de fonctionnement : branchement. Relevé des angles, retours et soudures.

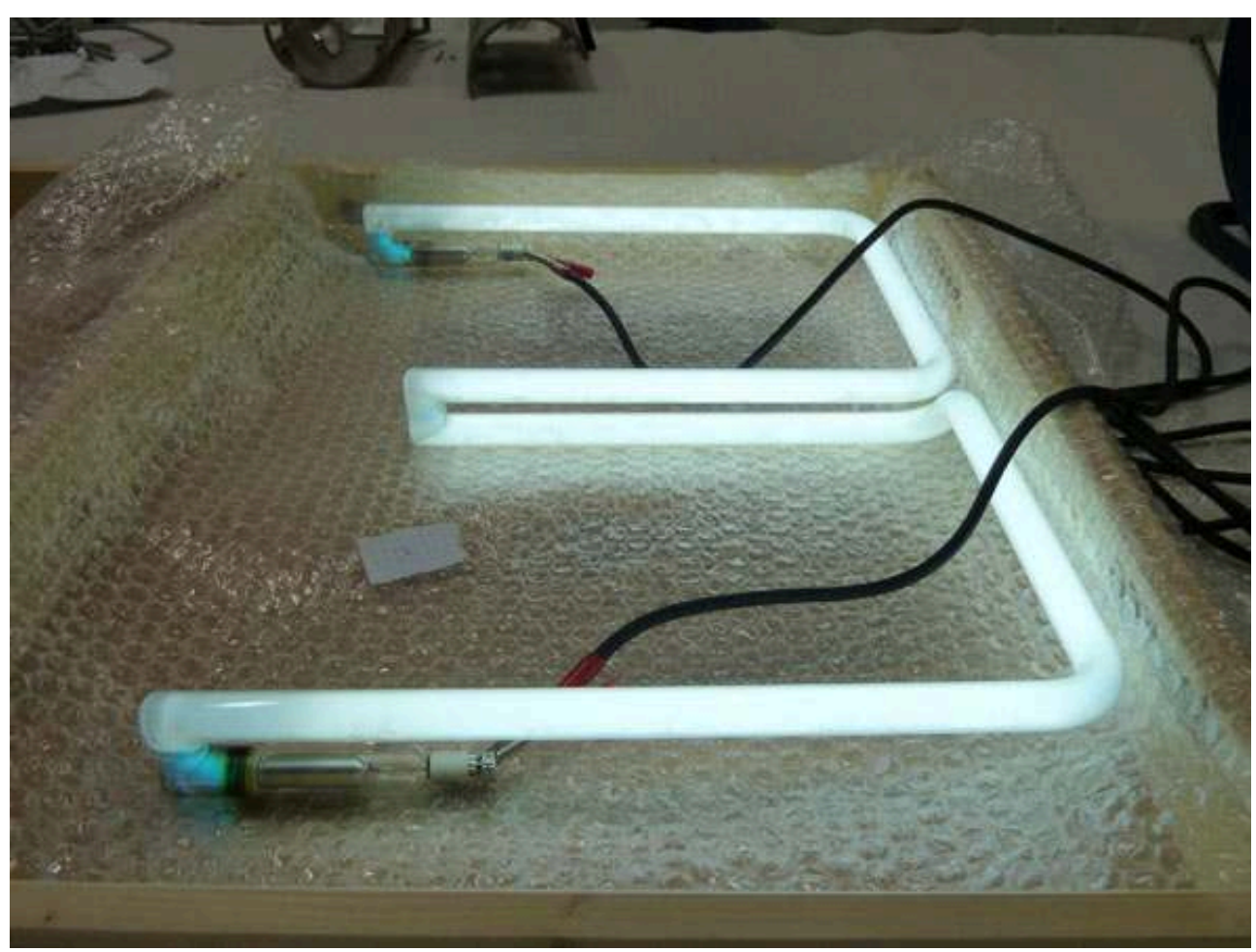

(c) Cécile Dazord, C2rmf 
Détail : électrode.

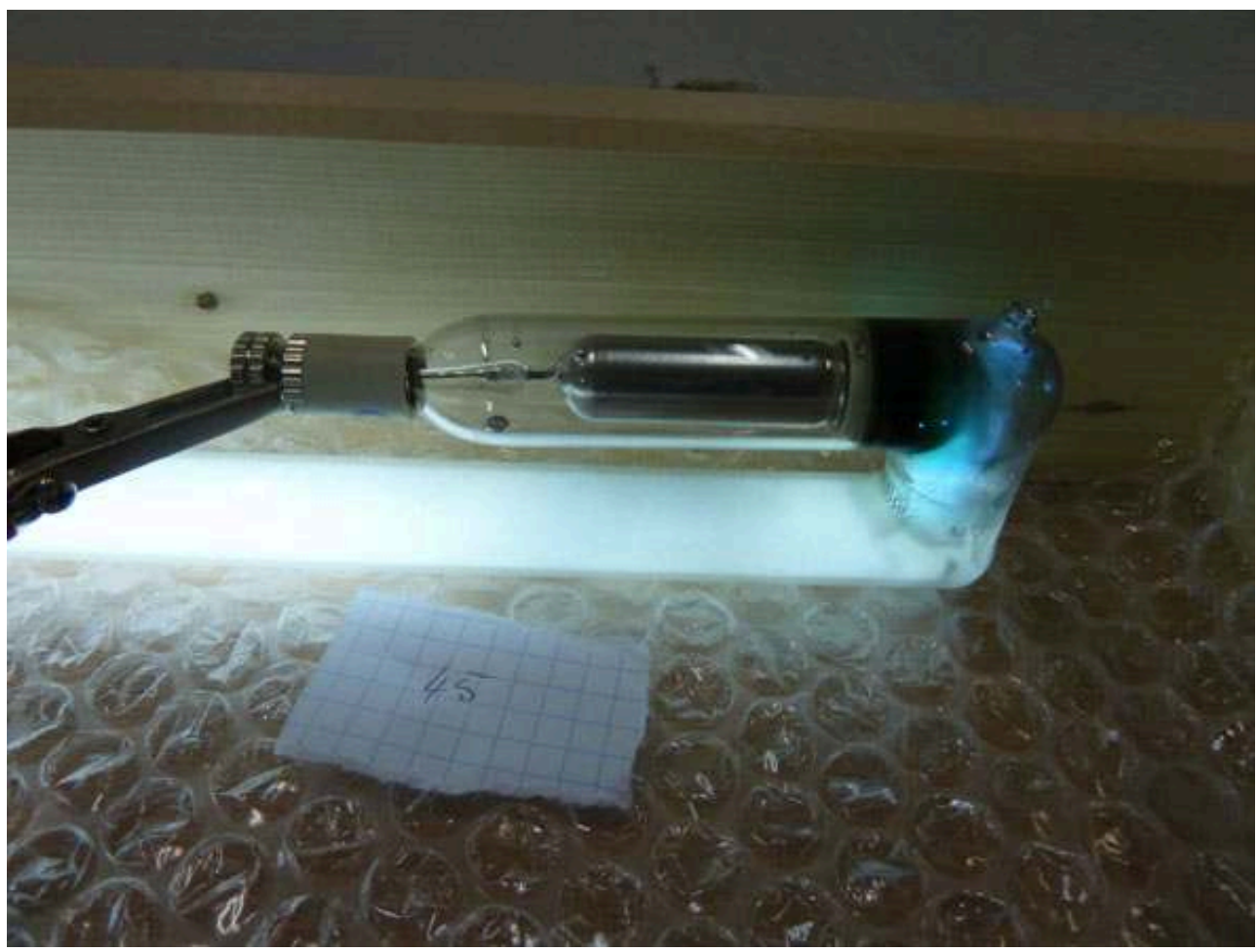

(C) Cécile Dazord, C2rmf

Détail : référence fabricant et référence couleur.

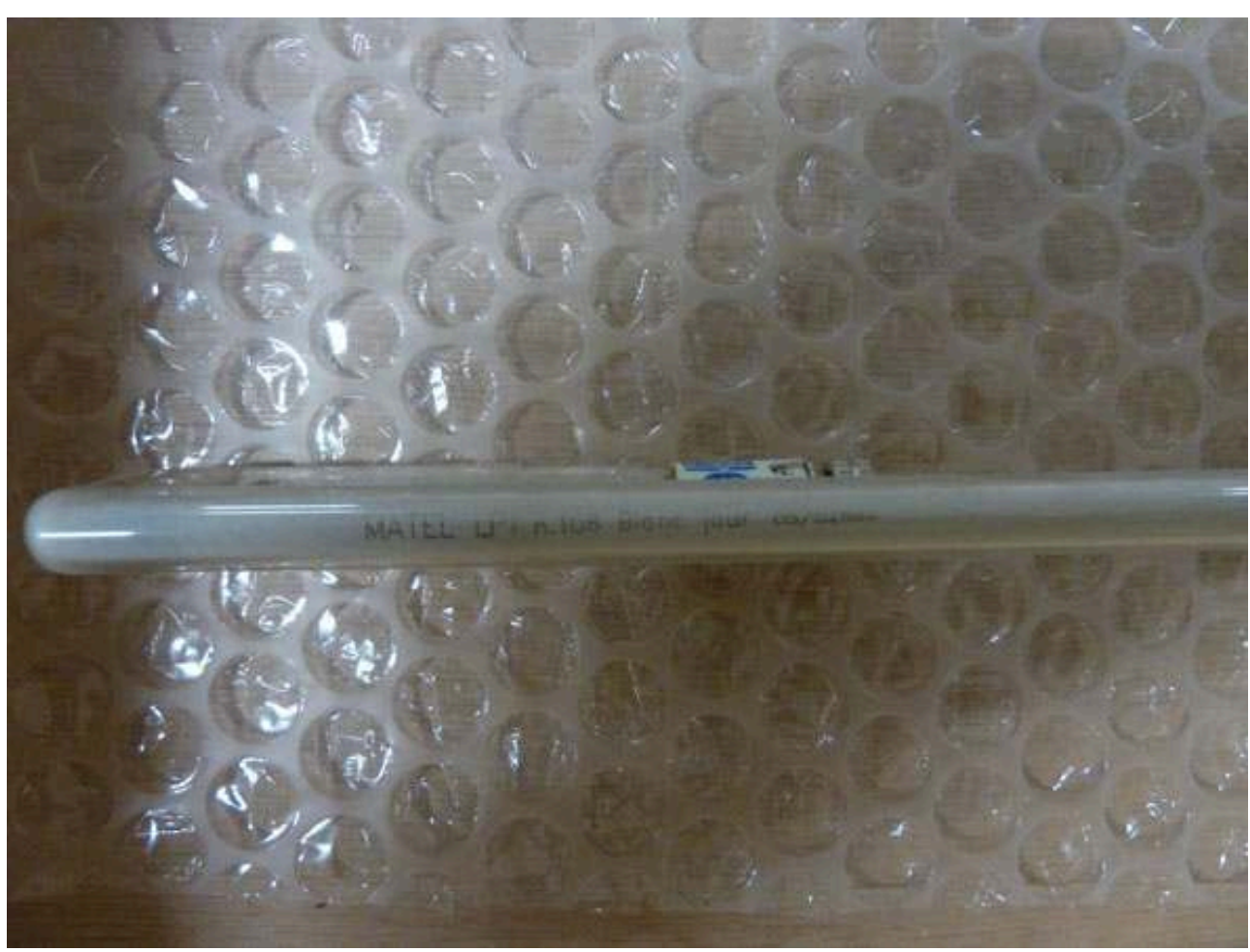

(c) Cécile Dazord, C2rmf 
17 Si l'appréhension des beaux-arts est majoritairement dominée par le paradigme indiciaire et une représentation de l'œuvre comme procédant rigoureusement d'une intuition antérieure ${ }^{5}$, ce schéma résiste mal à l'apparition dès les débuts $\mathrm{du} \mathrm{xx}^{\mathrm{e}}$ siècle d'objets en série dans les œuvres. Il résiste toutefois et si l'intention n'est plus logée dans la matérialité de l'œuvre elle peut être invoquée comme parole et consignée par écrit - on retrouve étonnamment ou plutôt significativement le binôme logos/écriture et l'affirmation d'une précédence du langage emblématique du paradigme sémiotique pour Derrida. Pour peu que l'on fasse entrer dans l'analyse des œuvres la prise en compte de la technique et de son rôle éventuellement contraignant ou significatif, constructif et producteur de sens, l'œuvre se prête alors à une analyse qui échappe au paradigme indiciaire. Si l'on prend pour exemple une œuvre de Joseph Kossuth - artiste emblématique de l'Art conceptuel - intitulée Néon, présentée dans l'exposition consacrée au « Néon dans l'art de 1940 à nous jours » en 2012 par la Maison rouge à Paris, elle consiste a priori en une inscription au néon du mot néon, une entité tautologique ne renvoyant à rien d'autre qu'à ce qu'elle donne à voir - sinon, précisément, que l'œuvre réalisée ne donne rien à voir (énonciation, en creux, de la seule validité du concept). Une observation technique plus précise montre la présence dans l'œuvre d'éléments habituellement utilisés en fabrication mais absents du produit achevé : le "queusot", tube de verre de $6 \mathrm{~mm}$ de diamètre ou au moyen duquel sont introduits le gaz et éventuellement le mercure nécessaires, et les bouchons de liège (aujourd'hui de silicone), employés par les néonistes lors du soufflage pour boucher une extrémité du tube. Quant au mot "néon", loin d'être redondant, il constitue davantage une antiphrase. On a coutume de désigner de manière simplificatrice et métonymique ce type de sources luminescentes à cathodes froides sous haute tension comme "néon ", mais le néon n'entre pas dans la fabrication de toutes les sources que l'on désigne ainsi abusivement. Certains néons ne contiennent pas de néon. C'est notamment le cas de celui de Kossuth dont la couleur blanche est issue de l'argon - employé seul, le néon produit une lumière rouge ; en aucune manière il ne peut produire de lumière blanche. Cet exemple montre clairement l'apport que peut constituer une étude technique précise des œuvres saisies comme "traces", prises dans un jeu de renvois multiples "entre tous les aspects de l'activité humaine " (ibid: 10) selon la formule de Benjamin évoquant Arago et la photographie. Dans le domaine de l'histoire des arts une telle approche semble avoir vu le jour notamment chez Moholy-Nagy et Benjamin ou plus tard chez Kracauer et prit racine dans une analyse des procédés photographiques et filmiques et de leur impact sur les œuvres d'art. On peut conjecturer que la place et le rôle de la technique doivent être envisagés à partir de la photographie et du film et, plus encore, à partir de la conscience immédiate et aiguë de certains penseurs témoins et contemporains de l'ascension de ces nouvelles formes d'expression nées avec et de la technique et l'industrie - du bouleversement de paradigme qui devait immanquablement en résulter. 


\section{BIBLIOGRAPHIE}

Anders, G. 2002 [1956] L'Obsolescence de l'homme. Sur l'âme à l'époque de la deuxième révolution industrielle, Paris : Ivrea, Encyclopédie des nuisances.

Arasse, D. 1992 Le Détail, Pour une histoire rapprochée de la peinture, Paris : Flammarion Idées et recherche.

Benjamin, W. 2012 [1938] Petite histoire de la photographie, Paris : Allia.

- 2010 « La Tâche du traducteur » (1923), in Euvres 1, Paris : Gallimard (Folio essai).

- 1991 [1936] « L'œuvre d'art à l'époque de sa reproduction mécanisée », in Écrits français, Paris : Gallimard (Bibliothèque des idées).

Castelnuovo, E. «Attribution », Encyclopédie Universalis.

Derrida, J. 2009 [1967] La Voix et le phénomène, Paris : PUF (Quadrige).

- 1967 De la grammatologie, Paris : Éditions de Minuit (Collection critique).

Eco, U. 2010 [1990] Les Limites de l'interprétation, Paris : Grasset (Le livre de Poche).

Ginzburg, C. 2010 [1986] Mythes, emblèmes et traces, Lagrasse : Verdier poche.

Goodman, N. 1980 [1968] Langages de l'art. Une approche de la théorie des symboles, Paris : Jacqueline Chambon (Rayon art).

Koldehoff, S., Timm, T. 2013 L'Affaire Beltracchi, Arles : Actes Sud.

Krauss, R. 1993 [1977] « Notes sur l'index » in Le Mythe de l'avant-garde et autres mythes modernistes, Macula : 65-92.

Michaud, É. 1997 Fabriques de l'homme nouveau : de Léger à Mondrian, Paris : Éditions Carré (collection Art \& esthétique).

- 1987 « La Vie moderne (Bauhaus et modernisme) », Cahiers du Musée National d'Art Moderne: 19-20 « Moderne, modernité, modernisme », Paris : Musée National d'Art Moderne : 130-135.

Moholy-Nagy, L. 1993 Peinture, photographie, film (1925) et autres écrits sur la photographie, Paris : Jacqueline Chambon.

Passuth, K. 1980 Moholy-Nagy et Walter Benjamin, Une rencontre. Cahiers du Musée national d'art moderne : 5 : 398-409.

Slade, G. 2006 Made to break, Technology and Obsolescence in America, Harvard University Press.

Urrichio, W. 2004 « Historicizing media in transition », in Rethinking media change, David Thornburn and Henry Jenkins ed., Cambridge, London : MIT Press.

\section{NOTES}

1. Jugée en 2011 l'«Affaire Beltracchi » a révélé la production, la mise en circulation sur le marché de l'art et l'acquisition par des institutions prestigieuses d'un nombre considérable de toiles d'artistes parmi les plus fameux du début du $\mathrm{xx}^{\mathrm{e}}$ siècle.

2. Le Musée d'art moderne et contemporain de Strasbourg a acquis en 2002 un diaporama photonumérique sonore vidéoprojeté de Nicolas Moulin intitulé Viderparis et numéroté $1 / 3$. Les deux 
autres occurrences sont rigoureusement identiques; Le même musée a produit en 2003 une installation de Cidlo Meireles eurêka/Blindhotland (constituée de 2001 boules en caoutchouc de poids différent de 500 à $1500 \mathrm{gr}$ ), d'un filet délimitant l'espace d'un revêtement au sol, d'une balance et d'une pièce sonore. La réédition de cette installation réalisée en 1975 puis disparue a été conçue comme une occurrence, deux autres pouvant être réalisées ultérieurement, chacun de ces trois exemplaires chacun prenant pour dominante une couleur différente (beige, noire et rouge).

3. L'entretien avec les artistes est le leitmotiv de toutes les initiatives initiées depuis le début des années 2000 pour doter la conservation-restauration de l'art contemporain d'une déontologie et méthodologie. Des guides d'aide à la réalisation d'entretiens sont publiés: Cf. http:// www.incca.nl/files/pdf/resources/guide_to_good_practice.pdf.

4. L'exposition Kunstlicht aus Lichtkunst présentée au ZKM en 2005 abordait notamment le sujet à travers un article rédigé dans le catalogue par Peter Weibel.

5. Certaines esthétiques prennent à rebours ce parti pris dominant. Ces le cas notamment de Konrad Fiedler (1841-1895) qui met en avant le «faire pour voir»; ou encore, plus récemment, de Luigi Pareyson et la théorie de la « formativité » (1918-1991).

\section{RÉSUMÉS}

Une caractéristique de l'œuvre d'art est qu'elle procède de deux ordres, l'intelligible et sensible, dont l'articulation conditionne la possibilité et les modalités d'une connaissance de l'œuvre d'art et de l'œuvre d'art comme vecteur de connaissance. Comment les beaux-arts traditionnels, fondés sur l'expression ou la traduction d'une intuition ou d'un concept dans un matériau ou une forme, puis, à partir du début $\mathrm{du} \mathrm{XX}^{\mathrm{e}}$ siècle, sur des pratiques artistiques marquées par l'irruption et l'intégration d'objets produits industriellement en série, s'accommodent-ils de cette hétérogénéité sans se dissoudre dans l'extériorité du matériau ou de l'objet sériel?

Envisagé comme indice actuel et circonstancié d'un donné originaire chez Ginzburg ou dépourvu, au contraire, de toute fonction indiciaire chez Derrida, le concept de trace constitue un point de départ à partir duquel repenser le rapport entre l'intelligible et le sensible dans l'œuvre d'art et notamment la place et le statut des objets produits industriellement en série. Il s'agit, en d'autres termes, à partir du concept de trace - et de ce qui permet de le confondre avec l'empreinte, le signe, le symbole ou, au contraire, de l'en distinguer - de reconsidérer l'articulation entre l'art et la technique.

An important aspect of a work of art is how it functions in two senses: the sensible and the intelligible - the articulation of the two is the condition for recognizing said work of art as an object of knowledge and as a vehicle for knowledge. How do fine arts - traditionally based on the expression of an intuition or a concept into a material shape and, since early 20th century, often integrating industrially-mass-produced objects - cope with this heterogeneity without vanishing into the exteriority of the material or the serial object?

Considered as a current and appropriate index of an original given according to Ginzburg or, on the contrary, devoid of any indexical function according to Derrida, the concept of "trace" stands as a starting point from which the distinctions between the sensible and the intelligible in the artwork - and more specifically, the status of mass-produced objects - can be re-examined and re-evaluated. In other words, the aim is, by the means of the concept "trace" - in so far it can be 
merged with the notions of print, sign or symbol or on the contrary be differentiated from them - to reconsider the articulation of art and technics.

INDEX

Keywords : intelligible, sensitive, art and technique, impress/mark

Mots-clés : intelligible, sensible, art et technique, traces

\section{AUTEUR}

CÉCILE DAZORD

Département recherche / Art contemporain, Centre de recherche et de restauration des musées de France (C2RMF) 